\title{
On the Optimal Adhesion Control of a Vortex Climbing Robot
}

\author{
Andreas Papadimitriou $^{1}$. George Andrikopoulos ${ }^{1}$ (D) . George Nikolakopoulos ${ }^{1}$
}

Received: 24 May 2020 / Accepted: 11 May 2021 / Published online: 3 June 2021

(c) The Author(s) 2021

\begin{abstract}
This article tackles the challenge of negative pressure adhesion control of a Vortex Robotic (VR) platform, which utilizes a modified Electric Ducted Fan (EDF)-based design for successfully adhering to surfaces of variable curvature. The resulting Vortex Actuation (VA) system is estimated through a switching Autoregressive-Moving-Average with eXternal input (ARMAX) identification, for accurately capturing the throttle to adhesion force relationship throughout its operating range. For safe attachment of the robot on a surface, the critical adhesion is modeled based on the geometrical properties of the robotic platform for providing the required reference forces. Within this work, an explicit controller via the use of a Constraint Finite Time Optimal Control (CFTOC) approach is designed in an offline manner, which results in a lookup table realization that ensures overall system stability in all state transitions. In an effort to further improve the tracking response for arbitrary setup orientations, the adhesion control scheme is extended through a switching EMPC framework. The resulting frameworks are tested through both dynamic simulation and experimental sequences involving: a) adhesion control on a rotating test curved surface and, b) adhesion and locomotion sequences on a water pipe.
\end{abstract}

Keywords Climbing robots $\cdot$ Switching modeling $\cdot$ Adhesion control $\cdot$ Model predictive control

\section{Introduction}

In the last decade, robotic applications have steadily gained interest in the maintenance field of large infrastructures [1]. Often, the aging and remote infrastructure, that it is difficult or dangerous to be reached by human inspectors, could be accessed by inspection robots that are operating in autonomous or semi-autonomous modes. Maintenance tasks, such as thickness measurement, visual surveillance, coating, brushing, etc. are a few examples of inspection and repair applications that could be performed by such robotic

This work has received funding from the European Union's H2020 Framework Programme under the call FET-OPEN, grant agreement No. 665238.

George Andrikopoulos andrikopg@gmail.com

Andreas Papadimitriou andpap@1tu.se

George Nikolakopoulos geonik@1tu.se

1 Luleå University of Technology, Luleå, SE-97187, Sweden platforms [2]. Wall climbing robots [1, 3-7] and their potential ability of accessing hazardous and unreachable by humans work environments makes them a good candidate for autonomously handling various inspection and maintenance tasks, in industries like construction [8,9], aviation [10-12], energy [13, 14], marine [15], etc.

\subsection{Background and Motivation}

In response to the need of improving inspection and maintenance quality, personnel safety, while reducing the overall cost and time, a novel Vortex Robot (VR) [16] has been proposed to autonomously perform inspection and repair tasks via interchangeable sensor and tool modules. As part of the FET-OPEN H2020 project "CompInnova", the target-specific goal is to utilize the platform for performing autonomous inspection of composite airplane structures via infrared-thermography and phased-array sensing modules Fig. 1. There are several key problems needed to be addressed in the related studies, provided that the target surfaces are curved. The major one being the ability of the robot to remain attached on the inspected aircraft structure, independent of its curvature and robot's orientation, while being able to handle high payloads for transferring the tools and sensor modules to the area of interest. 


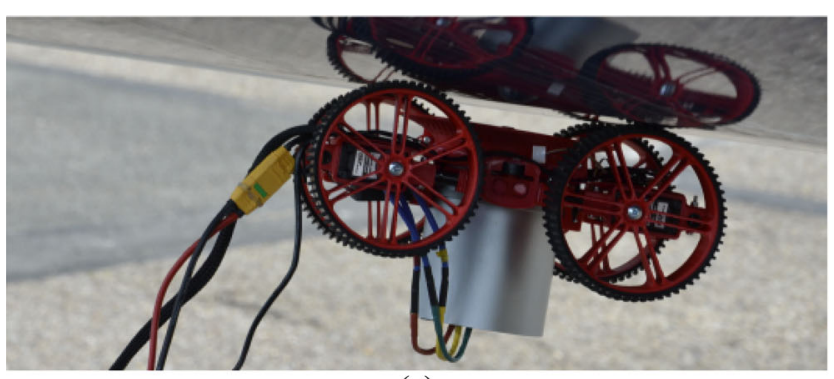

(a)

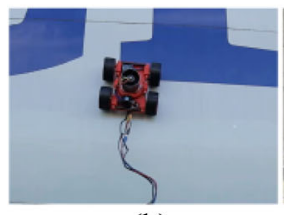

(b)

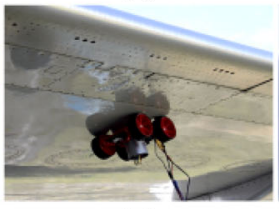

(c)

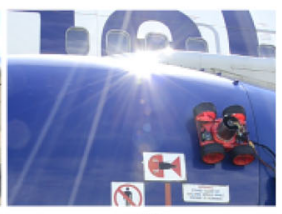

(d)

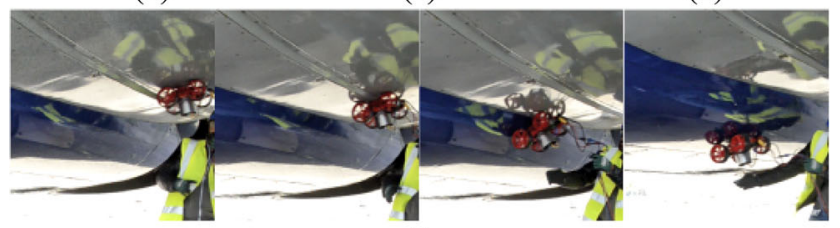

(e)

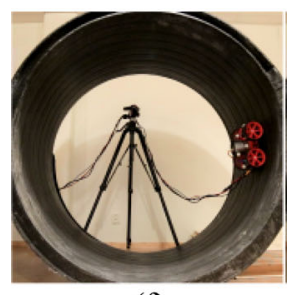

(f)

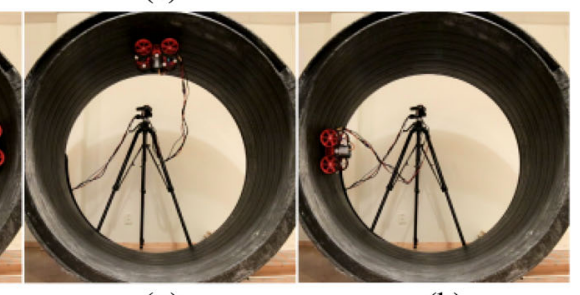

(g)

(h)
Fig. 1 Photographic stills of the VR while attached and moving under varying orientations and speeds on the surfaces of a-h Cranfield Boeing 737: a-b fuselage, $\mathbf{c}$ wing, $\mathbf{d}$ turbine engine $\mathbf{e}$ fuselage to wing transition, and $\mathbf{f}-\mathbf{h}$ the interior of a water drainage pipe

For handling such tasks, a climbing robot needs to maintain manoeuvrability, sufficient adhesion and high payload to weight ratio to successfully accomplish given inspection tasks. The vast majority of climbing robots, independently of the adhesion method (magnetic, pneumatic, gripping etc), are teleoperated under on-off-based adhesion controllers [12, 17], without being able to effectively control the level of adhesion. Only a few attempts found in the related literature, have addressed the issue of controlled adhesion for wall climbing robots [6, 14, 18-22], thus highlighting the need for advanced control schemes in order to minimize power consumption while satisfying all demanding operation requirements.

In general, during the inspection and maintenance procedures, the adhesion performance can be optimized based on various variables, such as the type of surface, orientation, equipment weight etc. To this purpose, the adhesion technology selected for the VR platform, hereafter referred to as Vortex Actuation (VA) [23], utilizes a modified Electric Ducted Fan (EDF) design to maintain adhesion on both flat and curved surfaces of the airplane, while maximizing the generated adhesion force, without requiring contact with the surface. Given the generally high maximal energy consumption of commercially available EDFs, the development of an energy efficient controller is deemed of paramount importance.

While the VR moves along the fuselage of the airplane, the inclination of the platform and airplane's curvature varies, thus introducing the need for an adhesion model that will be able to adapt to the alternating force requirements for supporting its weight and payload. In addition, mechanical inaccuracies and disturbances are leading to changes on the Center-of-Mass (CoM) that sequentially affect the overall performance of the utilised control scheme, with authors' previous efforts being focused on solving this problem from a static force and payload perspective [24].

Model Predictive Control (MPC) scheme is an optimal model-based control strategy that has been quite popular in the process industry [25] in the last decades, while in recent years there has been a major shift for its adoption in the area of robotics [26-28]. Among its advantages, MPC is capable of controlling MIMO systems that usually have strong couplings, while it can also handle constraints on the states, outputs, input rate etc. A characteristic of MPC is the ability to give predictions for future values of the system's variables, which is utilized from the controller to solve an online optimization problem.

The optimization process is repeated at every iteration, for selecting the best input sequence that will drive the system to the desired reference, while the complexity of this calculation depends on various characteristics, such as the complexity of the model, the prediction and control horizon, the number of optimization variables, constraints, etc. This repeated task is considered to be computational intensive [29] and quite often there is a need for expensive computation units to handle this type of problems. A strategy to overcome the challenge of extended computation power of the MPC is to design the controller in an off-line manner. The concept of Explicit-MPC (EMPC) is to calculate in advance the optimal solution of the MPC optimization problem as a Piece-Wise Affine (PWA) function, which effectively reduces the computation time and increases the controller's applicability.

\subsection{Contributions}

The contributions of this article are multiple. First, the VA system is estimated as an Autoregressive Moving Average Exogenous (ARMAX) model, for accurately describing the throttle to adhesion force relationship, while incorporating the additive disturbances from the force sensor measurements. To adequately address the hysteretic characteristics induced by the Electronic Speed Controller 
(ESC) and the EDF, experimentally acquired input/output data of different operating regions are utilized for an offline estimation of a linear model set, which are later incorporated in the model-based control.

Second, a switching EMPC structure is proposed for optimal regulation of the VR's adhesion. The explicit controller is implemented via the MPT toolbox [30] and it is applied on the case of a model-based static force computation of the required adhesion for given robot 2DOF orientation. The efficiency of the proposed control framework is initially evaluated under simulation trials, where the VR is dynamically tested on a set curved surface resembling the dimensions of an Airbus A350 fuselage.

Finally, the proposed control framework is experimentally evaluated. A single model EMPC is first tested for the case of VR immobilization on a composite curved surface with similar characteristics of an airplanes' surface, and while on alternating inclinations. Lastly, the performance of a switching EMPC is evaluated on a moving VR while placed on a cylindrical surface to demonstrate its applicability and efficiency in regulating the adhesion during simultaneous locomotion control for a given reference path.

\subsection{Outline}

The rest of this article is structured as follows. In Section 2, the modeling of the adhesion properties and motion of the robot are presented. Section 3 provides the theoretical framework for the VA identification, while Section 4 presents the EMPC control synthesis, with additional description of the locomotion control structure used in the evaluation sequences. The model identification sequences, as well as the closed-loop system evaluation under simulation and experimental trials, are presented in Section 5. Finally, concluding remarks, discussion points of the robots operability and limitations, as well as future work are discussed in Sections 6 and 7, respectively.

\section{VR Modeling}

\subsection{Critical Adhesion Modeling}

Figure 2 highlights the main components of the VR platform. The robot is a differentially steered platform actuated by four smart servo-motors, capable of provided continuous rotation with speed and torque control. The incorporated VA is composed of three main elements: a) the modified EDF [23] for the generation of the adhesion force, b) the ESC for driving the EDF, and c) the two load cells integrated between the EDF and the VR body for sensing the exerted force. The rest of the electronics including the load cell amplifier and the wifi-enabled microprocessing unit are properly integrated in the structure, while the robot
Fig. 2 Overview of the VR components

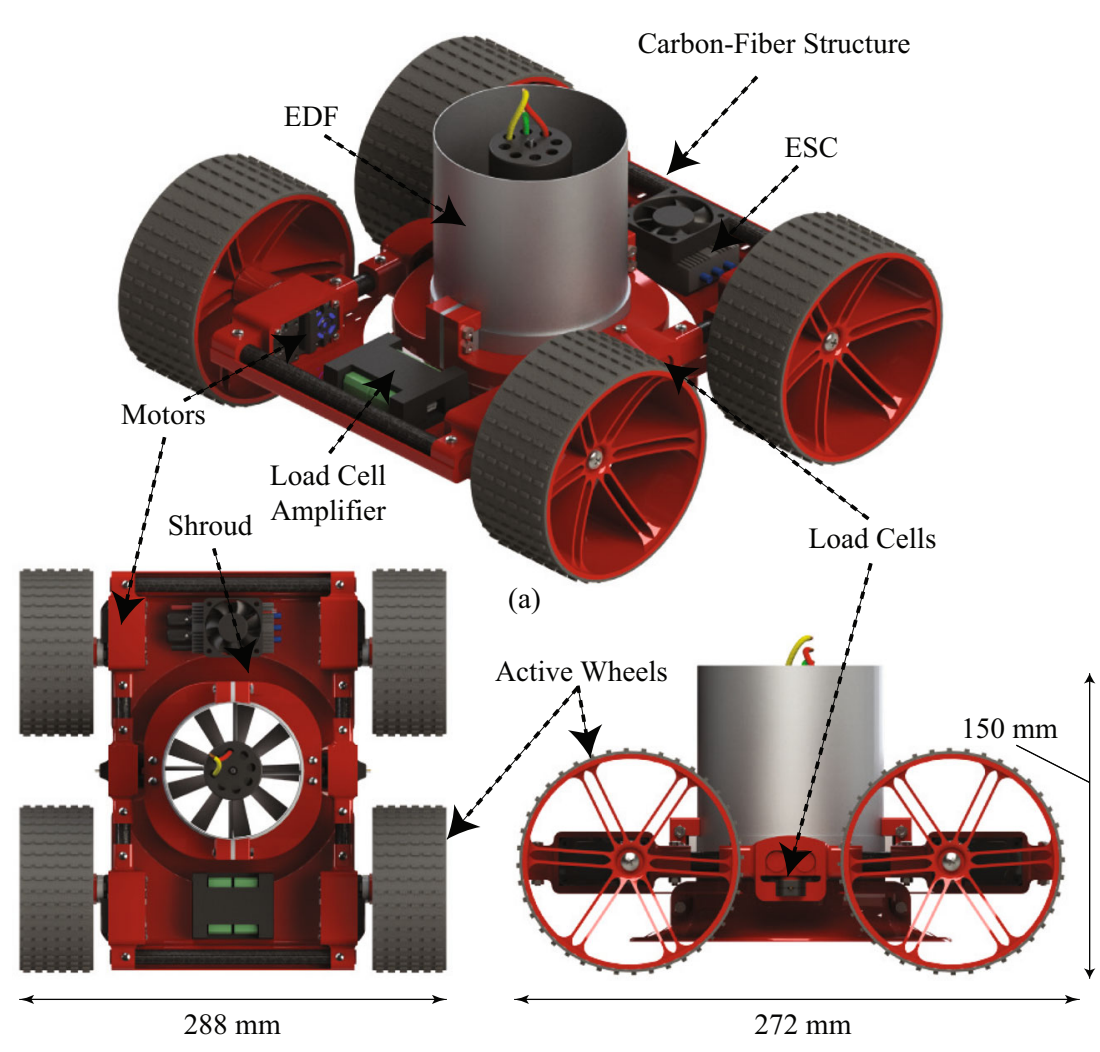

(b) 
prototype is powered through a tether cable via an external power supply unit.

Figure 3 depicts the VR with highlighted the main geometrical and kinematic elements. One of the main goals is modeling the critical force required for the robot to remain attached on a surface. Successful adhesion is considered when the robotic platform remains attached and immobilized i.e the angular velocity of the wheels $\omega_{i}=0$ for $i=\{1, \ldots, 4\}$ during inclination changes of the $\{\phi, \theta, \psi\}=\{$ roll, pitch and yaw $\}$ on the robot's frame $\{B\}$, with respect to the global frame $\{G\}$. Thus, the critical adhesion force $F_{\mathrm{C}}$ can be described by the below geometrical model [24]:

$F_{\mathrm{C}}=\frac{m g}{\mu} \sqrt{(s \phi)^{2}+(c \phi s \theta)^{2}}-m g c \phi c \theta$,

where $m$ is the mass of the system, $g$ is the gravitational acceleration, $\mu$ is the friction coefficient, while $\mathrm{c} \cdot \mathrm{s} \cdot$ denote the trigonometric functions $\cos (\cdot)$ and $\sin (\cdot)$, respectively. From Eq. 1 it can be noticed that the critical force $F_{\mathrm{C}}$ is independent from the rotation around the $z$-axis. Furthermore, below the critical angles, denoted as $\phi_{\mathrm{C}}, \theta_{\mathrm{C}}$, which are dependent on the friction and the mass of the platform, the described model (1) results into negative forces that are not feasible by the physical setup. Thus, the critical adhesion model is updated accordingly as:

$$
F_{\mathrm{C}}= \begin{cases}0 & 0 \leq \gamma<\gamma_{\mathrm{C}} \\ \frac{m g}{\mu} \sqrt{(s \phi)^{2}+(c \phi s \theta)^{2}}-m g c \phi c \theta & \gamma_{\mathrm{C}} \leq \gamma<\left(2 \pi-\gamma_{\mathrm{C}}\right) \\ 0 & \left(2 \pi-\gamma_{\mathrm{C}}\right) \leq \gamma \leq 2 \pi\end{cases}
$$

where $\gamma$ is defined as $\gamma=\sqrt{\phi^{2}+\theta^{2}}$ and $\gamma_{\mathrm{C}}=\phi_{\mathrm{C}}=\theta_{\mathrm{C}}$. In addition, the load cells used for measuring the force exerted from the VA, are able to sense the reaction force

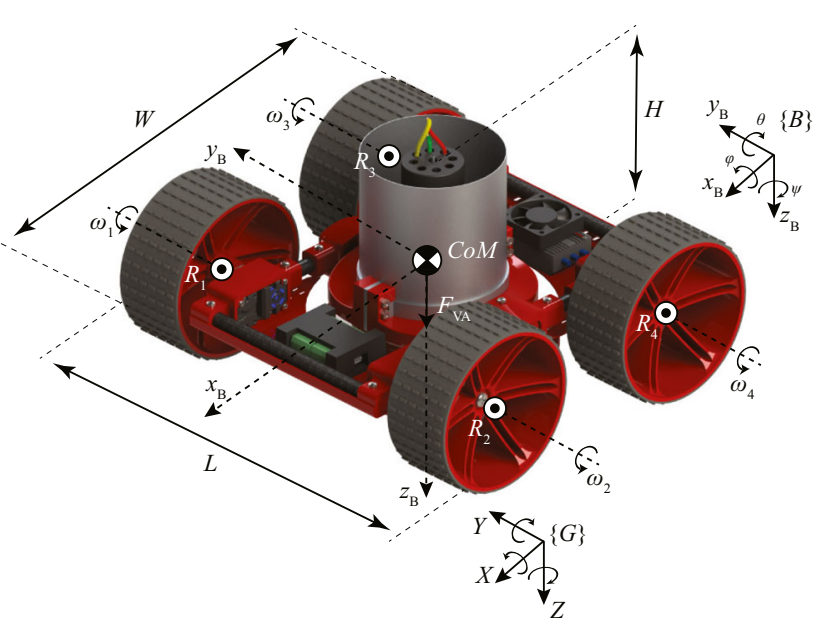

Fig. 3 Overview of the VR's DoF and main geometrical properties
$F_{\mathrm{S}}=\sum_{j=1}^{2} F_{\mathrm{S}, j}$, under the safe assumption that the load cells are the only contact areas between the EDF and the robot's frame. To translate the acquired force measurement of the load cells to adhesion force at the different plane orientations, the following conversion is used:

$F_{\mathrm{VA}}=F_{\mathrm{S}}-m_{\mathrm{VA}} g c \phi c \theta$,

where $m_{\mathrm{VA}}$ is the mass of the EDF and the supported components connected to the load cells. Under the additional assumption that the robot's geometrical properties do not alter significantly during motion, the presented critical adhesion model is also used when $\omega_{i} \neq 0$.

\subsection{Kinematic Analysis}

The VR is a differentially driven robot, which means the steering is based on the velocity levels of the left and right wheels. The local velocity of the body is assumed equal to the local velocity of the robot in $x$-direction in $\{\mathrm{B}\} v=$ $v_{x_{\mathrm{B}}}$, with the local velocity in $y$-direction being $v_{y_{\mathrm{B}}}=0$ since the robot cannot slip sideways [31]. The platform's configuration is represented by its coordinates $x_{\mathrm{B}}, y_{\mathrm{B}} \in \mathbb{R}$ and its orientation $\psi \in \mathbb{S}$ where $\mathbb{S}$ represents the set of unit circle. The angular velocity of the robot in the body frame $\{B\}$ is defined as:

$\dot{\psi}=\frac{U_{\mathrm{R}}-U_{\mathrm{L}}}{L}$,

where $U_{\mathrm{R}}, U_{\mathrm{L}} \in \mathbb{R}$ are the right and left wheel velocities, respectively, while $L$ is the platform's width. Under the assumption that the velocity of the left wheels is equal to each other, and the same applies to the velocity of the right wheels, the VR follows the kinematics of a unicycle model:

$$
\left[\begin{array}{c}
\dot{X} \\
\dot{Y} \\
\dot{\psi}
\end{array}\right]=\left[\begin{array}{cc}
\cos \psi & 0 \\
\sin \psi & 0 \\
0 & 1
\end{array}\right]\left[\begin{array}{c}
v \\
\omega
\end{array}\right]
$$

where $v$ and $\omega$ are the linear and angular velocity respectively in $\{\mathrm{G}\}$.

\section{Vortex Actuator Identification}

For the purposes of this study, the VA model has been modelled as a black box with the use of polynomial-based identification. Considering the general SISO discrete time system, the VA can be described as an ARMAX model:

$$
A(q) y(k)=B(q) u\left(k-n_{d}\right)+C(q) w(k),
$$


where $y(k), u\left(k-n_{d}\right), w(k), q$ denote the output, input delayed by $n_{d}$, noise and backward shift operator, respectively. Finally,

$$
\begin{aligned}
& A(q)=1+a_{1} q^{-1}+\ldots+a_{n_{a}} q^{-n_{a}} \\
& B(q)=b_{1} q^{-1}+\ldots+b_{n_{b}} q^{-n_{b+1}} \\
& C(q)=1+c_{1} q^{-1}+\ldots+c_{n_{c}} q^{-n_{c}},
\end{aligned}
$$

where $n_{a}, n_{b}$ and $n_{c}$ are the orders of the system [32].

Initially the $n_{a, \max }, n_{b, \max }, n_{c, \max }$ and $n_{d, \max }$ are set to high enough values to ensure that the system has lower order than the maximum assumption. The $e_{\mathrm{MSE}}$ is defined as the MSE (Mean-Square-Error) between the responses of the simulated identified system and the actual system, while used as a performance indicator of the ARMAX identification. Two threshold values are also set $e_{\mathrm{MSE}}^{\min }, e_{\mathrm{MSE}}^{\max }$ and utilized for the selection of the orders. Thus, based on algorithm 1 the model that results to $e_{\mathrm{MSE}}^{\min } \approx e_{\mathrm{MSE}}$ is the selected candidate. High order models with $e_{\mathrm{MSE}}<$ $e_{\mathrm{MSE}}^{\min }$ are prone to model mismatches as more sensitive in identifying noise and vibrations as dynamics of the system.

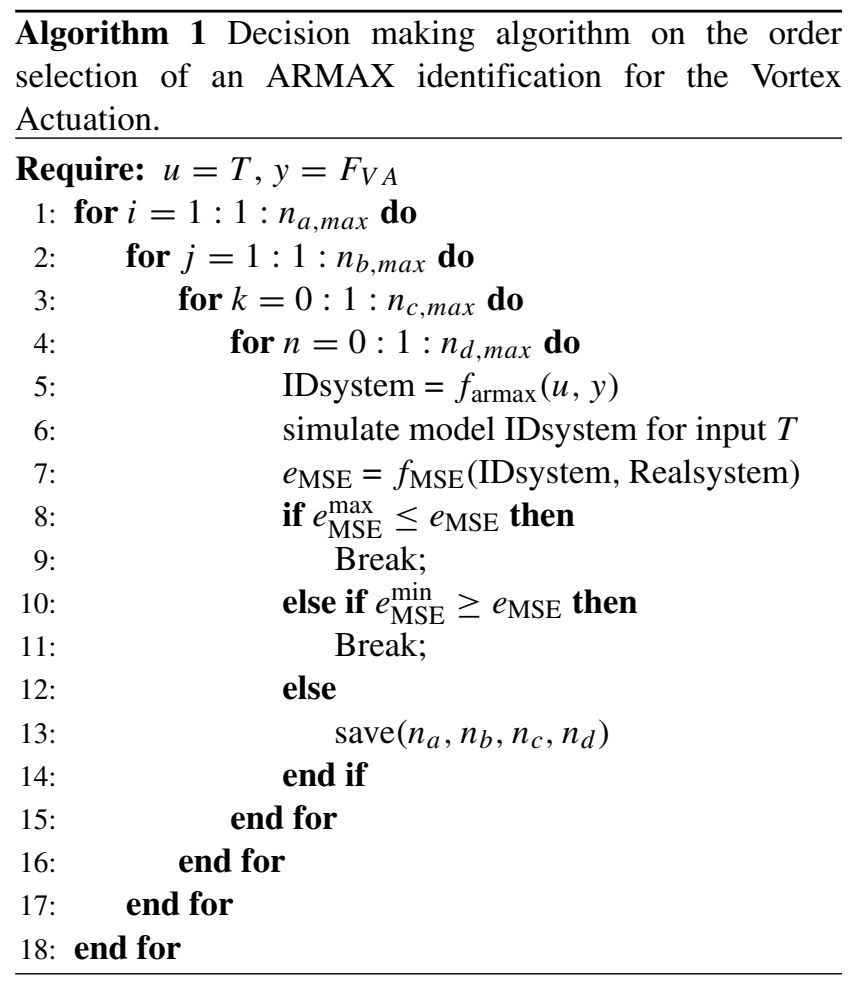

The identified linear discrete-time state space model is defined in Eq. 6 as:

$$
\begin{aligned}
x(t+1) & =\mathbf{A} x(t)+\mathbf{B} u(t) \\
y(t) & =\mathbf{C} x(t),
\end{aligned}
$$

where $x(t) \in \mathbb{R}^{p}$ are the $p$ states of the system, $u(t) \in$ $\mathbb{R}$ is the control input and finally $y(t) \in \mathbb{R}$ is the system output. The estimated matrices $\mathbf{A}, \mathbf{B}$ are expressed in their observable canonical form, while the parameters $a_{1}, \ldots, a_{n}$ and $b_{1}, \ldots, b_{m}$ are the identified parameters of the polynomial identification.

$$
\begin{aligned}
\mathbf{A} & =\left[\begin{array}{ccccc}
0 & 1 & 0 & \ldots & 0 \\
0 & 0 & 1 & \ldots & 0 \\
\vdots & \vdots & \vdots & \ddots & \vdots \\
0 & 0 & 0 & \ldots & 1 \\
-a_{n} & -a_{n-1} & -a_{n-2} & \ldots & -a_{1}
\end{array}\right], \\
\mathbf{B} & =\left[\begin{array}{llll}
0 & 0 & \ldots & 0
\end{array}\right]^{T}, \\
\mathbf{C} & =\left[\begin{array}{llll}
b_{m} & b_{m-1} & \ldots & b_{1}
\end{array}\right] .
\end{aligned}
$$

The described identification method results to the estimation of a linear system. For addressing the effect of hysteretic phenomena and noise disturbances induced by the actuating and sensing components of the adhesion system, a switching identification method is implemented for various operation regions.

Thus, a set of systems are extracted describing the system throughout its operating range. The identified transfer functions can be expressed as a piecewise model by a set of state-update and output equations, each valid in a polyhedral region of the state-space:

$$
\begin{aligned}
& x(t+1)=\left\{\begin{array}{l}
\mathbf{A}_{1} x(t)+\mathbf{B}_{1} u(t) \text { if } u(t) \in \mathcal{R}_{1} \\
\vdots \\
\mathbf{A}_{M} x(t)+\mathbf{B}_{M} u(t) \text { if } u(t) \in \mathcal{R}_{M}
\end{array}\right. \\
& y(t)=\left\{\begin{array}{l}
\mathbf{C}_{1} x(t)+\mathbf{D}_{1} u(t) \text { if } u(t) \in \mathcal{R}_{1} \\
\vdots \\
\mathbf{C}_{M} x(t)+\mathbf{D}_{M} u(t) \text { if } u(t) \in \mathcal{R}_{M}
\end{array}\right.
\end{aligned}
$$

\section{Control Synthesis}

\subsection{Switching EMPC Adhesion Control}

To optimally regulate the adhesion force generated from the $\mathrm{EDF}$, the following MPC problem is formulated:

$$
\begin{array}{cl}
\operatorname{minimize} & \sum_{k=0}^{N-1}\left(e_{F, t+k \mid t}^{\top} Q e_{F, t+k \mid t}+\Delta T_{t+k \mid t}^{\top} R \Delta T_{t+k \mid t}\right) \\
\text { subject to } & F_{\mathrm{VA}, \min } \leq F_{\mathrm{VA}, k} \leq F_{\mathrm{VA}, \max }, k=1, \ldots, N_{p}, \\
& T_{\min } \leq T_{k} \leq T_{\max }, k=1, \ldots, N_{p}, \\
& \Delta T_{\min } \leq \Delta T_{k} \leq \Delta T_{\max }, k=1, \ldots, N_{p}, \\
& e_{F, t+k \mid t}=F_{\mathrm{VA}, t+k \mid t}-F_{\mathrm{ref}}(t), k \geq 0, \\
& x_{t+k+1 \mid t}=f\left(x_{t+k \mid t}, T_{t+k \mid t}\right), k \geq 0, \\
& F_{\mathrm{VA}, t+k \mid t}=f\left(x_{t+k \mid t}\right), k \geq 0, \\
& T_{t+k}=T_{t+k-1}+\Delta T_{t+k}, k \geq 0, \\
& \Delta T_{t+k}=0, k \geq N_{p}
\end{array}
$$


which is solved at every time instant $t$, where $e_{F, t+k \mid t}$ denotes the predicted force error at time $t+k$, obtained by applying the input sequence $T_{t}, \ldots, T_{t+k-1}$ to Eq. 9 starting from the some initial conditions $x_{0}$ and $\Delta T$ is the input rate. Furthermore, $Q, R$ are the weight matrices of the optimization problem, while the constraints for the variables of the system are denoted as $(\cdot)_{\min , \max }$, with $N_{p} \in Z^{+}$to denote the prediction and control horizon.

The explicit controller receives the adhesion force error $e_{F}(t)$ and the previous input $T(t-1)$. The future control input vector $U(t) \in \mathbb{R}^{N_{p}}$ is derived from the EMPC and the next control action as first element of the input vector $T(t)=U_{1}(t)$. The measured force, from the load cells $F_{\mathrm{S}}$ is exerted from the EDF and it is filtered, based on the orientation of the robot at every iteration, to calculate the vertical component of the force. The force filtering results into the $F_{\mathrm{VA}}$, which indicates the adhesion level.

For comparison and evaluation purposes, this work considers two different state prediction models. Initially, a single linear discrete-time system (13) is considered, with the state predictions being

$f\left(x_{t+k \mid t}, T_{t+k \mid t}\right)=\mathbf{A} x_{t+k \mid t}+\mathbf{B} T_{t+k \mid t}$,

while in the case of the switching EMPC, we assume a set of PWA functions:

$f\left(x_{t+k \mid t}, T_{t+k \mid t}\right)=\left\{\begin{array}{l}\mathbf{A}_{1} x_{t}+\mathbf{B}_{1} T_{t} \text { if } x_{t}, T_{t} \in \mathcal{R}_{1} \\ \vdots \\ \mathbf{A}_{M} x_{t}+\mathbf{B}_{M} T_{t} \text { if } x_{t}, T_{t} \in \mathcal{R}_{M}\end{array}\right.$

For a given state vector $x_{k}$ the optimal solution is a PWA function of the form [33]:

$T_{0}\left(x_{0}\right)=\left\{\begin{array}{l}F_{1} x_{0}+G_{1} \text { if } x_{0} \in \mathcal{R}_{1} \\ \vdots \\ F_{M} x_{0}+G_{M} \text { if } x_{0} \in \mathcal{R}_{M}\end{array}\right.$,

where the parameter $F_{i}$ and $G_{i}$ are obtained from the MPT. $\mathcal{R}_{i}=\left\{x \mid H_{i} x \leq h_{i}\right\}$ are $M$ polyhedral critical regions obtained as well from the MPT [29]. The explicit solution of the optimization problem provides a look-up table, which converts the on-line optimization problem to a on-line sequential search algorithm.

The block diagram for the proposed adhesion control scheme is presented in Fig. 4. At each iteration the robot's orientation roll $\phi$ and pitch $\theta$ are assumed to be known and measurable. From the geometrical model, the critical adhesion $F_{\mathrm{c}}$ is calculated from Eq. 2 by utilizing the orientation feedback, robot's weight, payload and estimated friction between the wheels and the target surface. The reference force $F_{\text {ref }}(k)$ is calculated with the addition of a compensator gain $K \in[1,2]$ that accounts for the cabling load acting from the CoM of the robot, as well as any modeling inaccuracies. The EMPC receives the previous input $T_{t-1}$ and the force error $e_{F}$ to calculate the new $T_{t}$.

\subsection{Cascaded PI-Based Locomotion Control}

For the purposes of evaluating the VR under path-following scenarios, a PI-based control scheme was utilized, utilizing a PI controller for the velocity and a PI controller for steering control [16] as depicted in Fig 4. The purpose of this cascaded control scheme is to adjust the velocities of the left and right wheels, $U_{\mathrm{L}}, U_{\mathrm{R}}$ respectively, and lead to successful steering and desired velocity to enable the accurate tracking of the set trajectory way-points.

For a given reference point $p_{\text {ref }}=\left(x_{\text {ref }}, y_{\text {ref }}\right)$ in $\{G\}$ and the VR's current coordinates $p=(x, y)$ in $\{G\}$, the heading angle reference $\psi_{\text {ref }} \in \mathcal{R}$ is computed as shown in Eq. 16 . The heading error $e_{\mathrm{H}} \in[-\pi, \pi]$ is given in Eq. 17. The steering control effort $u_{\delta}$ is calculated from the PI controller and the $e_{\mathrm{H}}$ heading error.

$$
\begin{aligned}
\psi_{\text {ref }} & =\operatorname{atan}\left(\left(y_{\text {ref }}-y\right),\left(x_{\text {ref }}-x\right)\right) \\
e_{\mathrm{H}} & =\psi_{\text {ref }}-\psi
\end{aligned}
$$

The velocity reference value $v_{\text {ref }}$ is determined offline, while the speed is calculated from the euclidean distance covered from the robot at every time instant. Finally, the velocity error given by $e_{v}=v-v_{\text {ref }}$ is used from the second PI controller to calculate the the velocity control effort $u_{v}$. The left and right wheels velocities are computed by mixing the steering and velocity control efforts,

$$
\left[\begin{array}{l}
U_{\mathrm{L}} \\
U_{\mathrm{R}}
\end{array}\right]=\left[\begin{array}{cc}
1 & 1 \\
1 & -1
\end{array}\right]\left[\begin{array}{l}
u_{v} \\
u_{\delta}
\end{array}\right]
$$

\section{Evaluation Results}

\subsection{Identification Results}

The system identification has been performed under iterative experimental trials with the use of open-loop inputoutput data. The ARMAX identification has been performed for three different operating regions, for input data $T$ selected as $20-40 \%, 60-80 \%, 80-100 \%$ and the output data $F_{\mathrm{VA}}$ is $8-18 \mathrm{~N}, 32-50 \mathrm{~N}, 50-68 \mathrm{~N}$ respectively, while the orders of the resulting models have been fine-tuned to be $n_{a}=1, n_{b}=3, n_{c}=1$ and $n_{d}=1$.

For evaluating the model accuracy, the VA simulated response based on the identified models $F_{p}^{\text {model }}, p=\{1-3\}$ is presented in Fig. 5, against the experimental adhesion force acquired for an input throttle signal from $0-100 \%$ with $10 \%$ increments. From the presented results, $F_{1}^{\text {model }}$ 
Fig. 4 EMPC overall proposed control scheme for the adhesion regulation of the VR

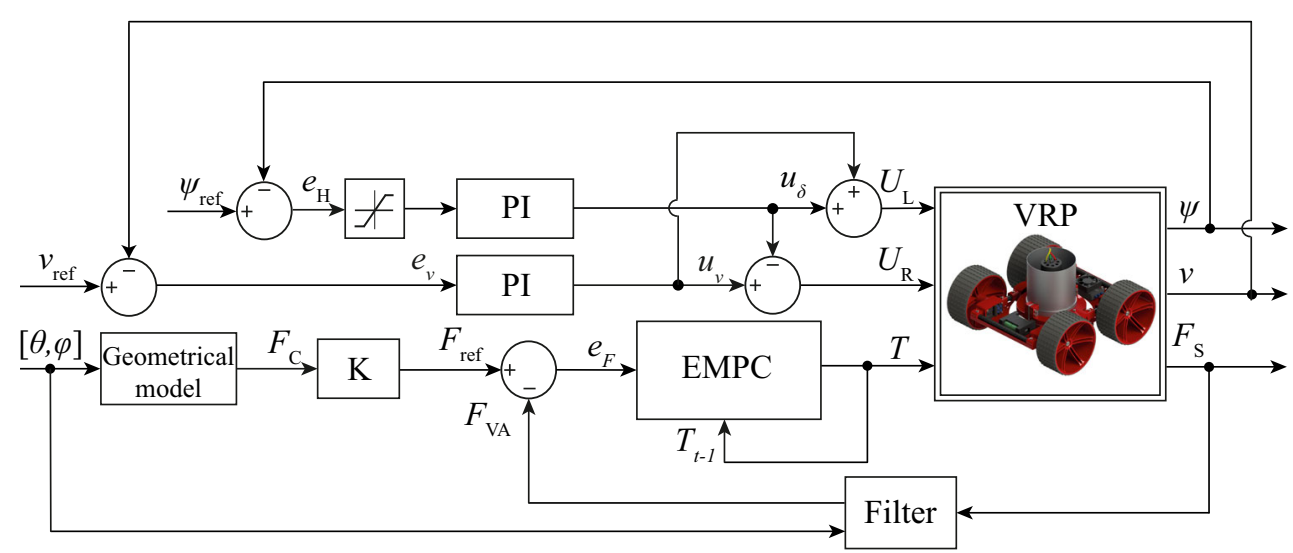

adequately describes the model only in the higher operating regions, $F_{2}^{\text {model }}$ provides better tracking of the output at an input $50 \% \leq T \leq 70 \%$, while $F_{3}^{\text {model }}$ has an overall high accuracy, with lower matches when the input to the system is in the region $0 \% \leq T \leq 40 \%$. These results are indicative of the nonlinear nature of the VA system [34, 35] and highlight the need for utilizing a PW model set for increasing the EMPC accuracy under all regions. The incorporation and testing of nonlinear models for further improving the overall identification accuracy is part of future work.

For the ARMAX identification of the switching EMPC, which was utilized in the experimental sequences, the system was split into five different operating regions. The input data $T$ selected as $0-40 \%, 40-50 \%, 50-60 \%$, $60-70 \%$ and $70-100 \%$ while the force $F_{\mathrm{VA}}$ is $0-18 \mathrm{~N}$, $18-24 \mathrm{~N}, 24-32 \mathrm{~N}, 32-41 \mathrm{~N}, 41-68 \mathrm{~N}$ respectively. The first and last function of PWA system have the largest operating regions $0 \leq u(k)<40$ and $70 \leq u(k)<100$ respectively, while each of the rest functions are valid only for a $10 \%$ of the total operating region of the VA.

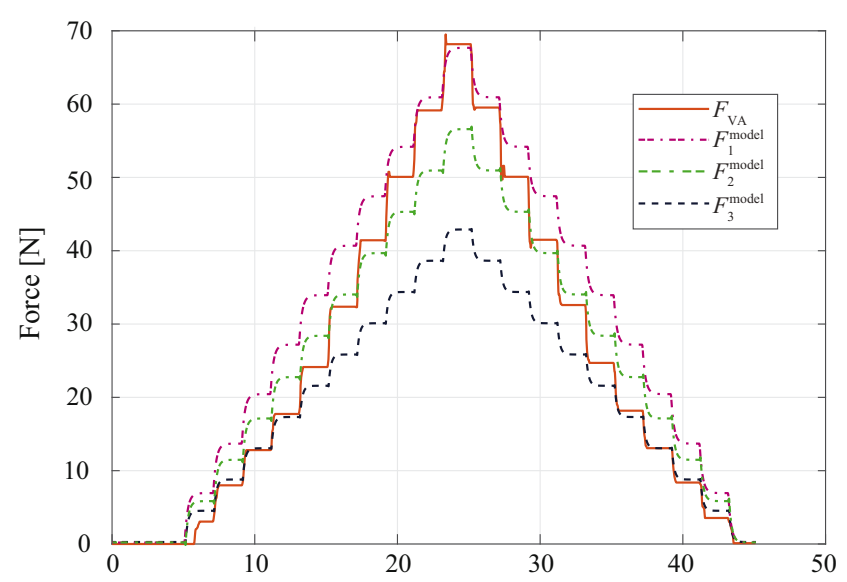

Fig. 5 VA identification results at different operating points

\subsection{Simulation Results}

To preliminary evaluate the efficacy of the overall proposed control scheme, the VR system underwent a series of simulation trials. For the needs of the presented evaluation, Matlab's and Simulink's Simscape Multibody [36] were used for dynamically simulating the setup's operation, utilizing the robot's CAD representation. The basic properties of the simulated VR platform utilized in the presented studies were $m=2.112 \mathrm{~kg}, \mu=0.8$, $F_{\mathrm{VA}, \max }=80 \mathrm{~N}, L=0.272 \mathrm{~m}, W=0.288 \mathrm{~m}, H=$ $0.150 \mathrm{~m}$ and $g=9.807 \mathrm{~m} / \mathrm{s}^{2}$, while the platform's CoM is extracted for the target materials set in the CAD design as $(x, y, z)=(-0.2,18.5,-1.4) \mathrm{mm}$ from its geometrical center. The test surface selected for the simulations is a cylinder with radius $r=3 \mathrm{~m}$ depicted in Fig. 6, resembling the maximal diameter of an Airbus A350 fuselage.

Finally, the simulated EMPC system was synthesized on the experimentally identified model set presented in the previous subsection. The EMPC output constraints have been set to $0 N \leq F_{\mathrm{VA}} \leq 68 \mathrm{~N}$ while the input is constrained at the operation region of ESC $0 \% \leq T \leq 100 \%$. The error $\Delta F_{\mathrm{VA}}$ of the reference point to the measured force has been penalized by a factor $Q=150$ and the input change has been penalized by $R=0.9$. The discrete time model had sampling time $T_{s}=25 \mathrm{~ms}$ and the resulted EMPC consisted of 25 regions.

Results from two simulation sets are presented, with the first having the platform completing a circular path around the test surface and under open-loop locomotion, for evaluating the adhesion control properties in all robot orientations. In the second set, the adhesion control on the most demanding orientation is evaluated, while the VR tracks a set path on the curved surface.

Figure 7 shows the orientation of the platform roll, pitch and yaw during the simulations. Both $\phi$ and $\psi$ angles remain at zero, which is indicative of the target heading during the simulation and the kinetic balance between 


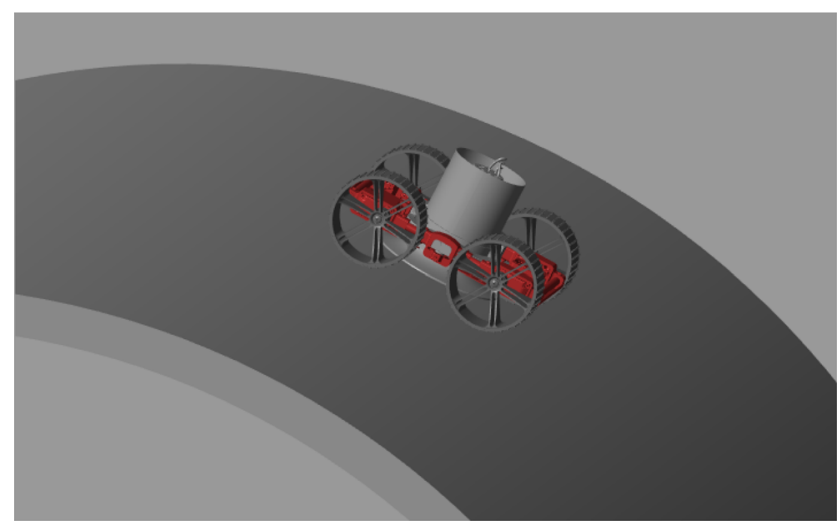

Fig. 6 Graphical overview displaying the VR while dynamically simulated in Simscape Multibody for the case of a cylindrical surface

the VR's left and right wheels, while $\theta$ varies between $\left(-180^{\circ}, 180^{\circ}\right)$ following the circles circumference.

The same figure presents the reference force $F_{\text {ref }}$ and the vortex adhesion $F_{\mathrm{VA}}$ response during the simulation, where the robot performed a full circular path around the cylinder's surface, with the bottom figure showing the control effort $T$. Between time instances $0-8$ seconds and $35-42$ seconds, where the inclination is approximately $-40^{\circ} \leq$ $\theta \leq 40^{\circ}$, the critical force $F_{c} \leq 0$ and results to $F_{\text {ref }}=0$ due to the orientation and weight of the platform as well as the friction between the surface and the wheels. When the VR's orientation is approximately at $\theta= \pm 130^{\circ}$, the critical adhesion and, consequently, the reference force reaches a peak value of $F_{\text {ref }}=53 \mathrm{~N}$, indicating the most demanding angle from an static adhesion point-of-view. At this demanding orientation the control effort is $T=76 \%$.

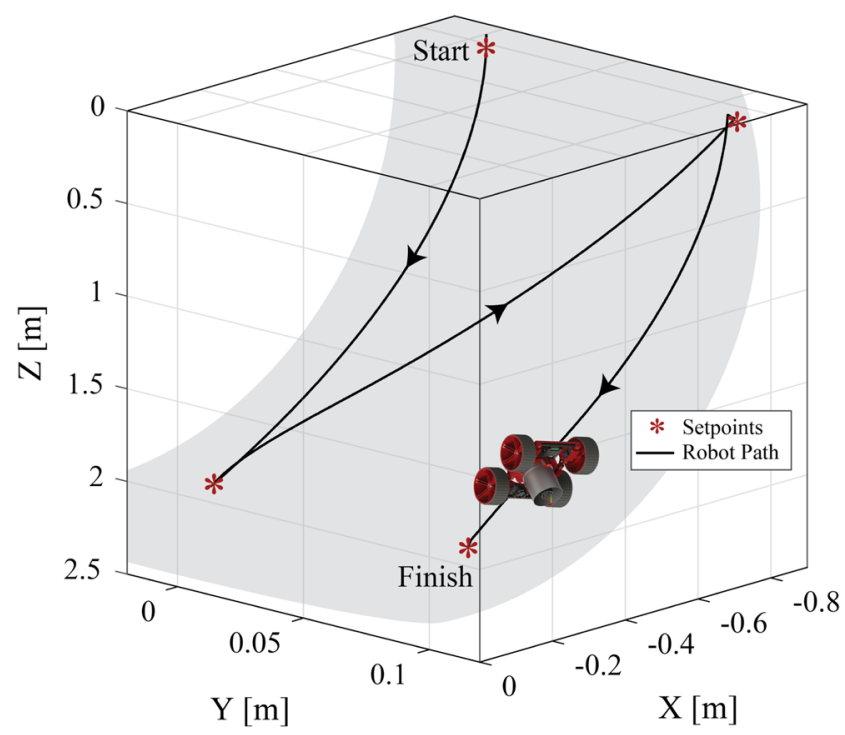

Fig. 8 VR's reference setpoints and dynamically simulated response, for the case where the VR is oriented at initially $\theta=90^{\circ}$ on a cylindrical surface of $1.5 \mathrm{~m}$ radius

Finally, when the robot is in the inverted pose $\theta= \pm 180^{\circ}$ the adhesion requirements are lower since the robot's weight is the only acting force. The overall RMS control error was calculated at $4.04 \mathrm{~N}$.

In the second simulation set, the VR's adhesion and locomotion performance is simulated for four given setpoint goals. Figure 8 presents the acquired response in comparison to the reference points. The locomotion algorithm is programmed to halt the motion of the VR when it reaches the point at a distance smaller than $8 \mathrm{~mm}$.
Fig. 7 (top) Reference $F_{\text {ref }}$ and adhesion force $F_{\mathrm{VA}}$ responses, (middle) throttle control effort $T$ response, and (bottom) the VR's roll, pitch and yaw acquired via dynamic simulation of a complete circular motion around a cylindrical surface of radius 3 $\mathrm{m}$
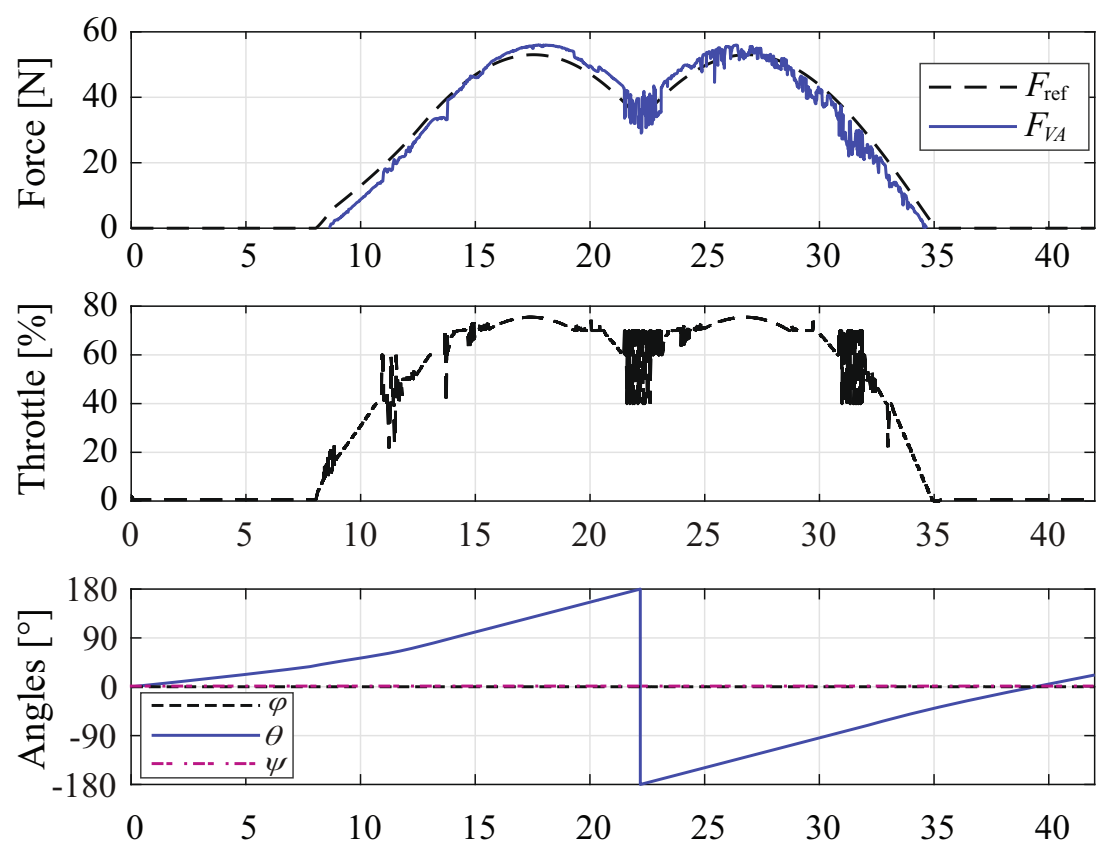
The acquired response is characterized by smooth motion and maneuverability, with the robot remaining attached on the surface at all times, while successfully tracking the reference points throughout the movement. The middle diagonal section of the response is tackled by the robot via backward motion, for avoiding large local yaw changes that could hinder the robot's performance on the cylindrical surface.

Figure 9 shows the angular responses for these reference points, where the initial orientation of the robot were selected as $\theta=90^{\circ}$, while the final orientation was $\theta=$ $130^{\circ}$. When the robot reaches the final point of a segment of the path, it remains immobilized on the surface at its current position, for the specific experiment this can be noticed at $\theta=90^{\circ}, 130^{\circ}$. When the robot steers diagonally on the curved surface it is noticeable an imbalance occurrence between the wheels and the surface, which appears on the pitch angle time response. As far as the adhesion response is concerned, Fig. 9 shows that the reference $F_{\text {ref }}$ varies between $42 \mathrm{~N}$ and $53 \mathrm{~N}$, which the EMPC manages to regulate successfully the adhesion force $F_{\mathrm{VA}}$ with the RMS error calculated at $3.16 \mathrm{~N}$.

\subsection{Experimental Results}

\subsubsection{Single Model EMPC}

For the evaluation of the EMPC scheme under the use of a single VA model, $F_{\text {model }}$ (Fig. 5) was selected as the candidate model because of its higher accuracy at the target operating regions, given the robot's properties and the nature of the planned experimental trials. The EMPC output constraints was set to $0 \leq F_{\mathrm{VA}}[\mathrm{N}] \leq 68$ while the input is constrained at the operation region of ESC $0 \leq T[\%] \leq 100$. The error $\Delta F_{\mathrm{VA}}$ of the reference point to the measured force was penalized by a factor $Q=2$ and the input change has been penalized by $R=0.01$. The discrete time model was formulated with sampling time $T_{s}=25 \mathrm{~ms}$ and the resulted EMPC consisted of 25 regions. The explicit controller was designed via the MPT toolbox, tuned via MATLAB and finally integrated to the VR platform as a C-code export.

For the experimental evaluation of the controller, a rotating platform with one DoF was utilized, which is depicted in Fig. 10a. A test surface of a Boeing 737 fuselage was attached on the manually rotated platform, while the VR adhesion capabilities were tested during the platform's immobilization states, $\omega_{i}=0$ for $i=1,2, \ldots, 4$. For the presented experimental trial, the close-loop performance was tested on two complete surface rotations in a clockwise and counterclockwise fashion for testing the adhesion efficiency. For the localization of the robot, the VICON motion capture system [37] has been utilized that provides the $x_{\mathrm{B}}, y_{\mathrm{B}}, z_{\mathrm{B}}$ position and the $\phi, \theta, \psi$ orientation feedback. The static friction between the wheels and the test surface was experimentally estimated at approximately $\mu=0.56$.

Photographic stills from the experimental evaluation are provided in Fig. 10 and $\mathrm{c}$ where the control scheme managed to keep the robot successfully attached on the test surface regardless of the surface orientations. In Fig. 11 the control
Fig. 9 (top) Reference $F_{\text {ref }}$ and adhesion force $F_{\mathrm{VA}}$ responses, (middle) throttle control effort $T$ response for a path following task while the robot is oriented at (bottom) $\theta=\left[90^{\circ}-130^{\circ}\right]$
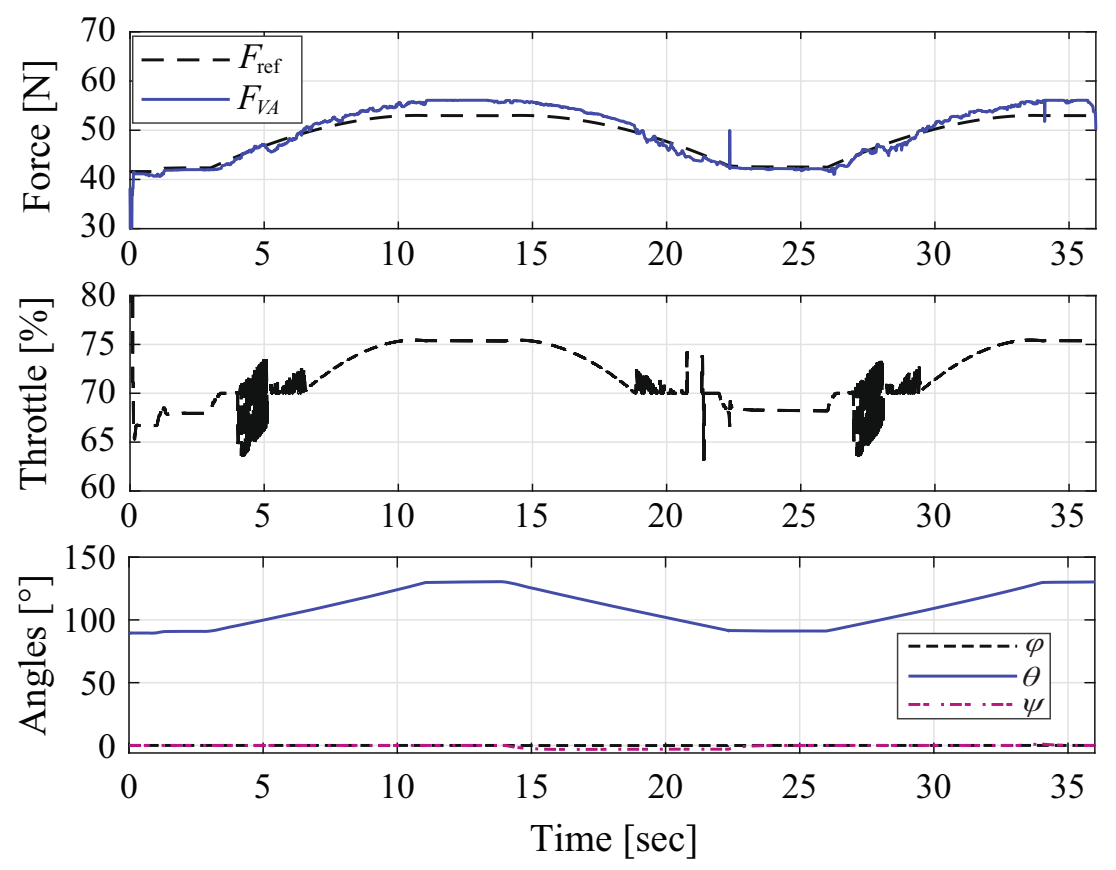


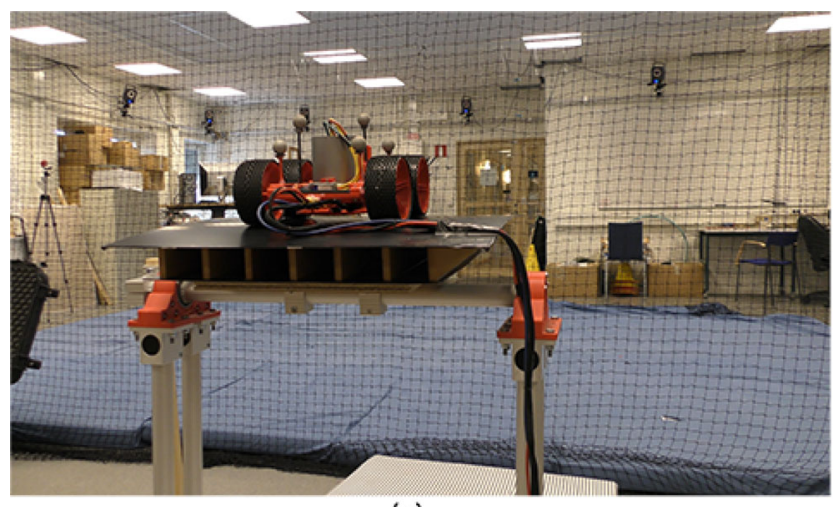

(a)

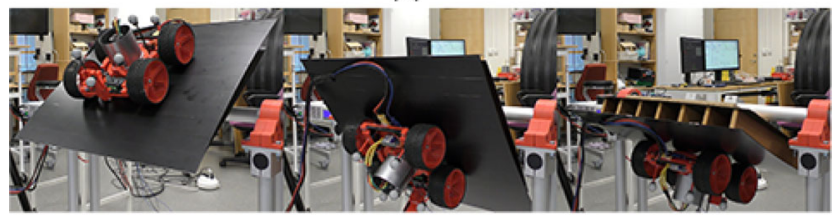

(b)

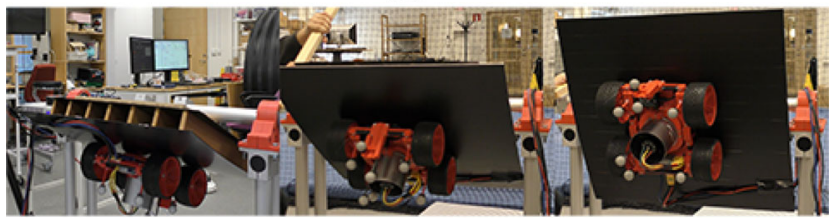

(c)

Fig. 10 Photographic stills showing the VR staying adhered while immobilized on a curved test surface, under alternating inclinations

performance of the EMPC is depicted. The generated force $F_{\mathrm{VA}}$ shows locally an accurate tracking of the reference force $F_{\text {ref }}$ around an $40-70 \mathrm{~N}$ operation region for which the controller has been designed, with the RMS error being calculated at $4.84 \mathrm{~N}$.

It has to be noted that the single model inaccuracy around the operating point of $0-40 \mathrm{~N}$, required the use of the force compensator $F_{c}$ with the gain tuned and set at $K=1.3$ in order for the overall scheme to provide sufficient thrust and reach the critical force levels. This can be observed when the pitch orientation is around $\theta=180^{\circ}$, where the additive constant compensator reaches the adhesion force to its maximum exerted force. These results further highlighted the need for a switching model for capturing the dynamic changes over the whole VA operating range, which would effectively reduce the model mismatches.

\subsubsection{Switching EMPC}

For the needs of experimentally evaluating the switching EMPC scheme, the control input was constrained in the operation region of the VA $0 \leq T \leq 100$, which are the physical limits of the ESC input signal. The output constraints were set to $0 \leq F_{\mathrm{VA}} \leq 68$, while the control horizon was set to $N_{c}=3$. The penalties for the error $\Delta F_{\mathrm{VA}}$
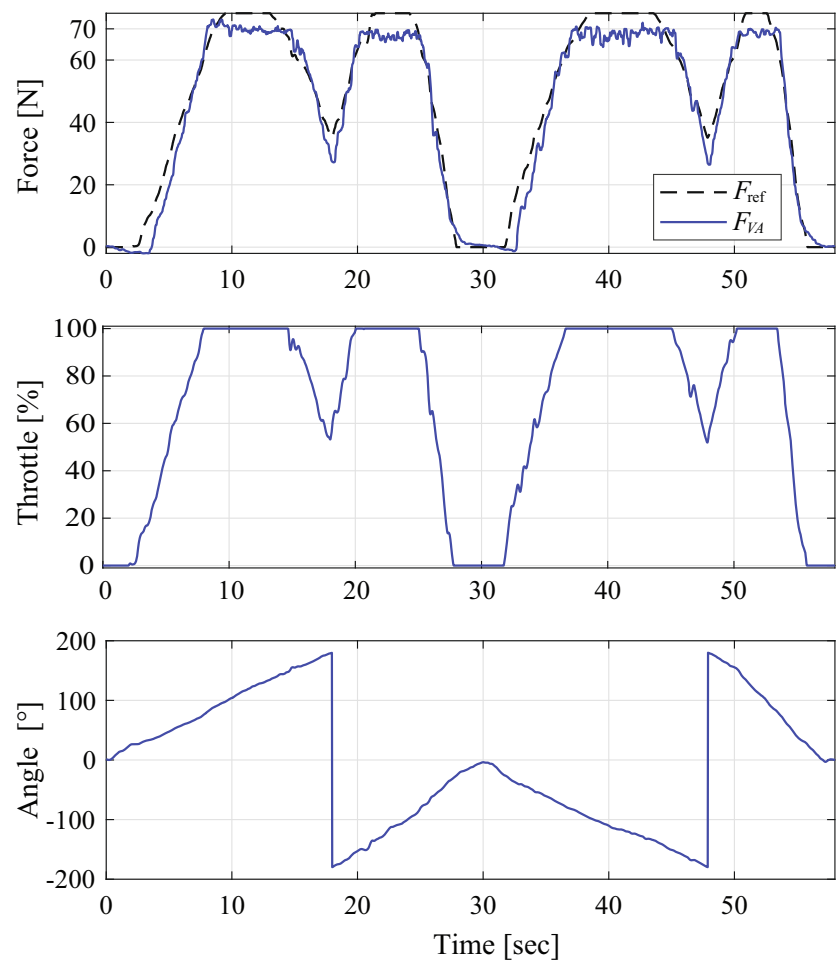

Fig. 11 Experimentally acquired data regarding the VR's adhesion properties for the single-model EMPC experimental trial. (top) The generated $F_{\mathrm{VA}}$ and reference force $F_{\text {ref }}$ responses, (middle) the throttle control effort response $T$, and (bottom) the pitch angle $\theta$ response

between the reference point and the measured force, and the input change $\Delta T$ were set at $Q=2$ and $R=0.3$, respectively.

For these experimental sequences, a water duct pipe of 1.6 meters diameter was utilized, as displayed in Fig. 12. The static friction between the wheels and pipe's surface was experimentally measured at approximately $\mu=$ 0.41 . These experimental trials targeted the performance evaluation of the adhesion controller during motion on a curved surface of varying inclination, which given the relatively small diameter and length of the pipe was increasing the experimental challenge. For that purpose, a linear reference path was selected and is graphically presented in Fig. 13, along with the acquired experimental response.

As far as the adhesion control is concerned, the target value $F_{\text {ref }}$ is computed at each iteration based on the robot's orientation and characteristics from Eq. 2. The explicit controller was able to successfully keep the force close to reference value as shown in Fig. 14. Since the robot moves in a straight line along the global x-axis, top Fig. 14, only the $\theta$ angle of the robot varies as it moves, while the $\phi$ angle remains as expected at approximately 0 degrees. Hence, it can be noticed how the reference value changes when the robot moves from $80^{\circ}$ to $-80^{\circ}$. The bottom Fig. 14 


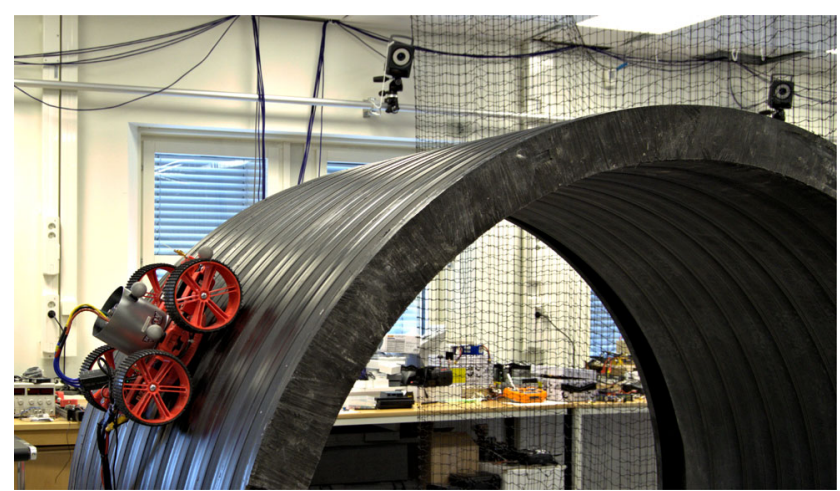

Fig. 12 Photographic still showing the VR operating on a water duct cylinder for the evaluation of the switching EMPC adhesion controller and the locomotion control for a given test path

shows the deviation $e_{F}$ of the adhesion force $F_{\mathrm{VA}}$ from the target value $F_{\text {ref }}$. The proposed EMPC controller is able to maintain the VR attached on the surface without slipping even on extreme angles, while the RMS error is calculated at $4.11 \mathrm{~N}$. Considering the load cell accuracy at $\pm 0.5 \mathrm{~N}$, the overall performance of the switching controller considered improved compared to the single model EMPC.

Figure 15 shows the power consumption $P$ and control action $T$, respectively. The maximum power for this experiment was approximately at $2400 \mathrm{~W}$. The power consumption follows similar response to the throttle. Finally, when the angle $\theta$ is approximately $\pm 40^{\circ}$ the control action jumps from $40 \%$ to $60 \%$ due to the tuning and the region change of the feedback law, while the adhesion force is not affected from these fast changes.

Figure 13 shows the given reference path and the actual robot response, while Fig. 16 shows the time responses for the heading error $e_{\mathrm{H}}$, velocity $v_{\text {actual }}$, and motors inputs $v_{m}^{\{\mathrm{L}, \mathrm{R}\}}$. From the obtained results, it can be observed that the

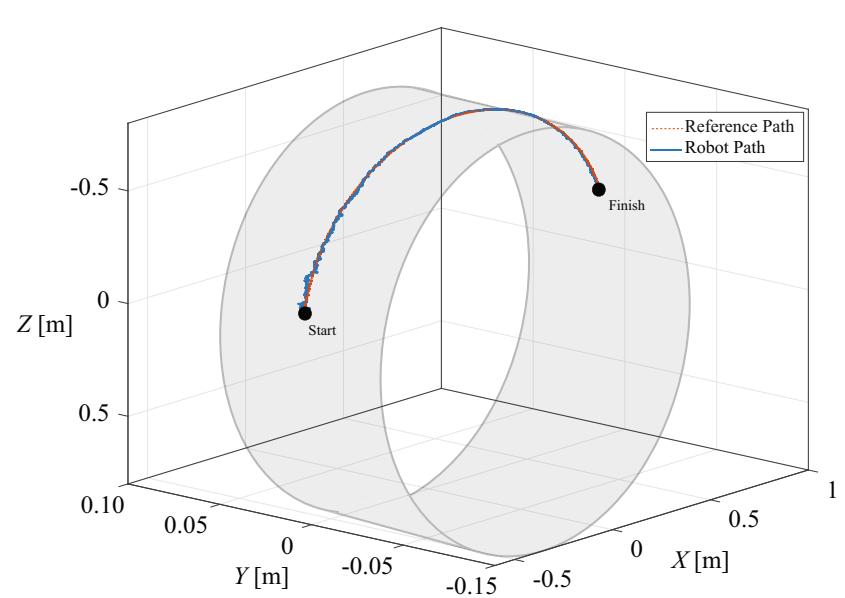

Fig. 13 3D representation of VR's path following response on a curved surface for the evaluation of the switching EMPC adhesion controller, for the case where the VR is oriented at initially $\theta=80^{\circ}$ on a cylindrical surface of $0.8 \mathrm{~m}$ radius
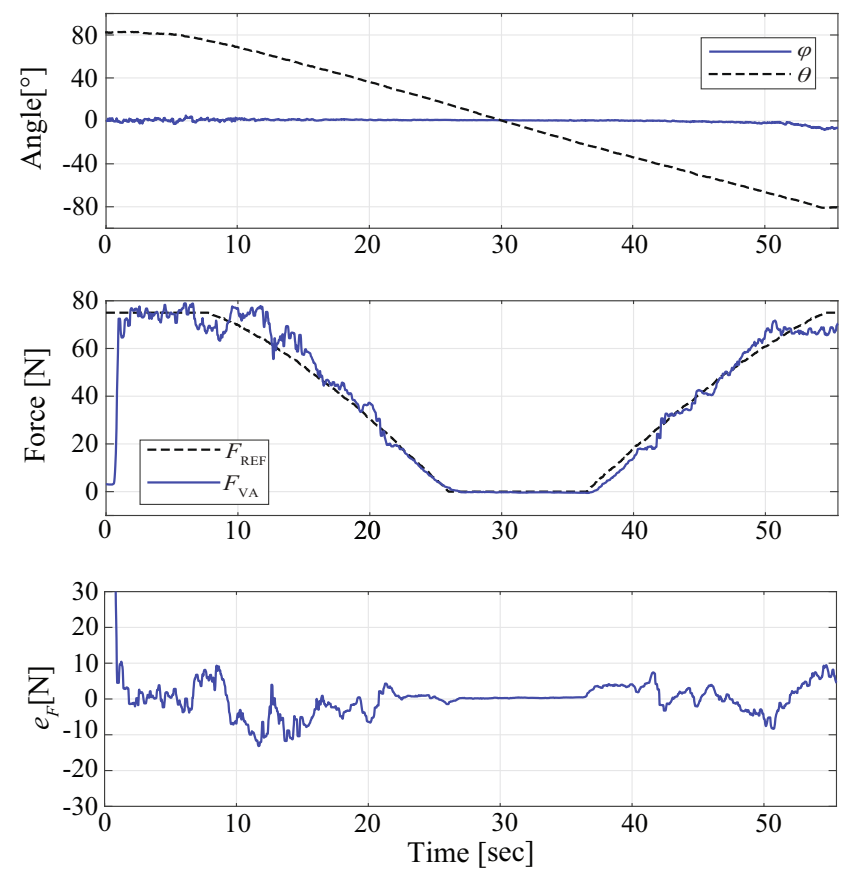

Fig. 14 (top) Time responses showing the three-axis orientation of the $\mathrm{VR}$, (middle) reference $F_{\text {ref }}$ and adhesion force $F_{\mathrm{VA}}$, (bottom) and the calculated force error $e_{F}$ for the switching EMPC experimental trial

heading error $e_{\mathrm{H}}$ remains under $\pm 0.1 \mathrm{rad}$ (top Fig. 16) for the total duration of the experiment. The velocity $v$ tracks the reference value at $0.04 \mathrm{~m} / \mathrm{s}$. It is noticeable that it takes approximately 7 seconds for the VR to accelerate and reach the target value (middle Fig. 16). This is happening due to the initial orientation of the robot, which is seen at $-80^{\circ}$, where the dynamic requirements are higher compared to the required ones when the robot starts at a horizontal plane.
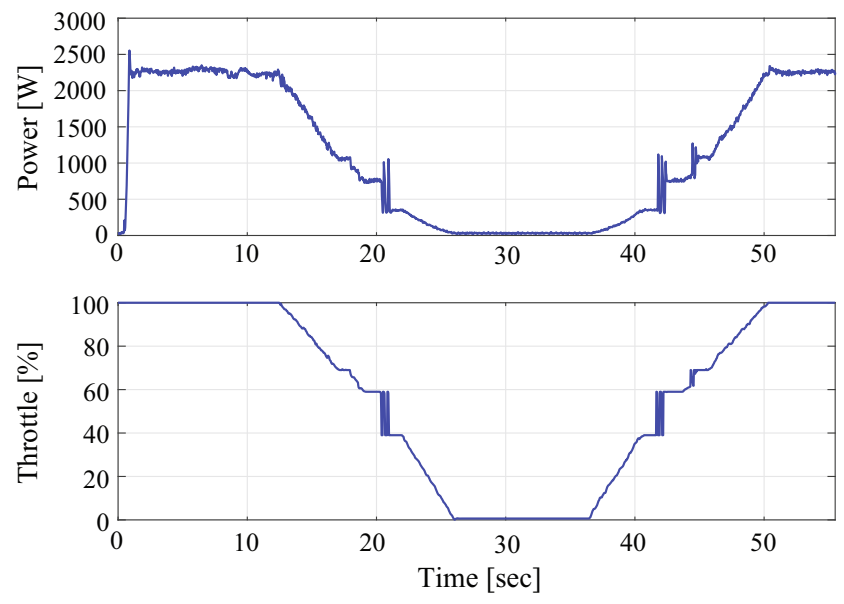

Fig. 15 Acquired responses of the VR power consumption $P$ (top) and throttle control action $T$ (bottom) for the switching EMPC experimental trial 

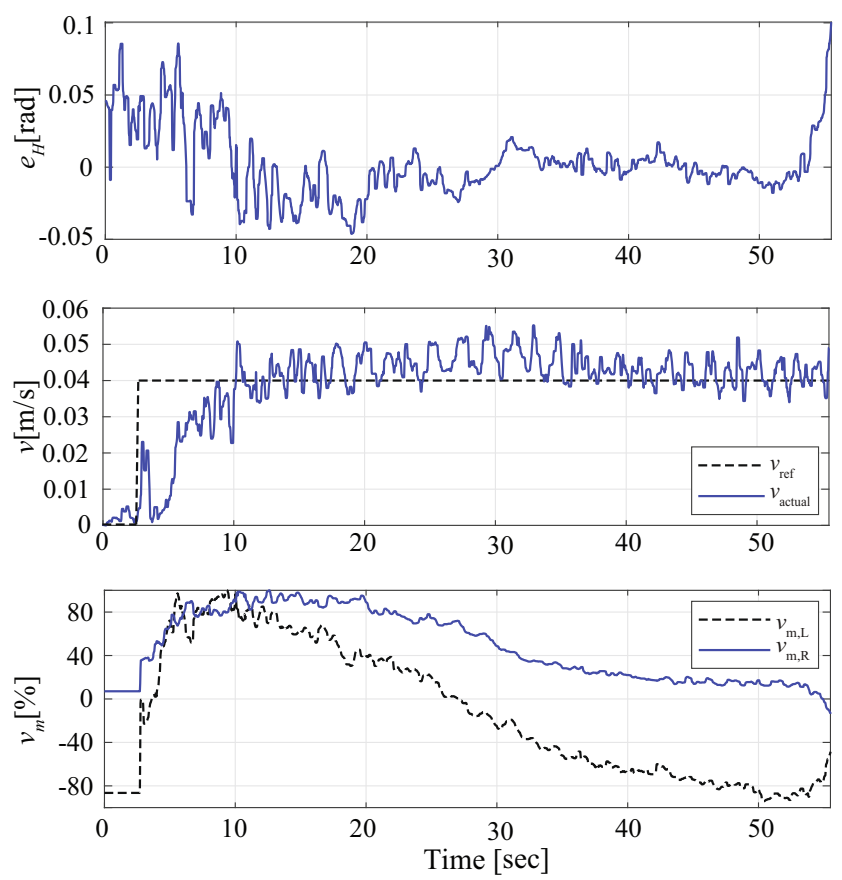

Fig. 16 Time responses of the locomotion components: in top the heading error $e_{\mathrm{H}}$, in the middle the velocity $v$, target velocity $v_{\text {ref }}$ and in bottom the motor input $v_{m L, R}$ are presented

It is also observed that when the pitch $\theta$ decreases and the robot starts to tilt forward, the motor inputs are decreasing to negative values for avoiding the acceleration increase due to the negative inclination.

\section{Conclusions}

In this article, the design and evaluation of an Explicit Model Predictive Control (EMPC)-based structure for regulating the adhesion level of a climbing Vortex Robot (VR) was presented. To enable the synthesis of such a model-based control scheme, the Vortex Actuation (VA) system was, first, approximated via a single ARMAX model through an identification sequence executed at a determined operation point. To better capture the nonlinearities induced by the modified Electric Ducted Fan (EDF) and Electronic Speed Controller (ESC) systems, a switching model approach was utilized, where the VA consisted of multiple PWA systems identified for different operation regions of the system. The VA model was then utilized as the base for the off-line synthesis of an EMPC scheme, an optimal control method capable of handling input/output constraints and ensuring system stability. The validity of the adhesion controller and its efficiency in controlling the adhesion force of the VR was evaluated through a series of simulation and experimental trials.
The closed-loop performance was first tested under dynamic simulations, with the CAD-imported robot model performing a full circular path around a test cylinder resembling the fuselage of an airplane. Utilizing a geometrical model for providing the critical adhesion reference to the EMPC, the VR system managed to successfully complete the circular motion with an RMS error of approximately $4.04 \mathrm{~N}$, with smooth adhesion and locomotion performance, showing that the EMPC was capable of providing smooth control efforts with absence of abrupt throttle changes. In addition, a setpoint-based path alternating between back and forth motions was also utilized for evaluating both the robots steering and adhering capabilities on the curved surface, which again provided successful system control with an adhesion RMS close to approximately $3.16 \mathrm{~N}$.

To further test the efficacy of the proposed scheme under single and PW model VA representations, experimental trials were performed involving a rotating test curved surface for immobilized evaluation of the EMPC-enabled adhesion, as well as trials on a water duct setup, where the VR utilized both the EMPC adhesion control and a PI-based cascaded locomotion controller for following a set path. The acquired results from the immobilized trials highlighted that the single model EMPC provided better results when the reference forces was close to the respective operating region with greater deviations around areas close to the operating minimum and maximum, thus providing a total RMS error of $4.84 \mathrm{~N}$. To address this, a switching EMPC was evaluated for the VR moving on the water duct. A drastical improvement was observed for the case of the switching EMPC, where the RMS adhesion error remained at approximately $4 \mathrm{~N}$.

In overall, the proposed switching EMPC approach manages to successfully control the adhesion for the VR prototype, while providing the important advantages of inherent system stability and the inclusion of MIMO systems, which makes it a suitable solution for future handling of both adhesion and locomotion performances.

\section{Discussion and Future Work}

Dynamic simulations over cases of different surface curvatures and orientations have shown that the prototype VR configuration has the ability to successfully traverse convex and concave surfaces of minimum $0.175 \mathrm{~m}$ and $0.215 \mathrm{~m}$ curvature radius Fig. 17, respectively. The VR operability over such cases and for a specific robot configuration, is affected by the loss of maximum adhesion force caused by the change in the distance between the surface and the VA shroud, which leads to a reduction of permissible payload. Nonetheless, the free-flight VA 
Fig. 17 Graphical representation of the minimum positive and negative curvatures supported by the current configuration of the VR

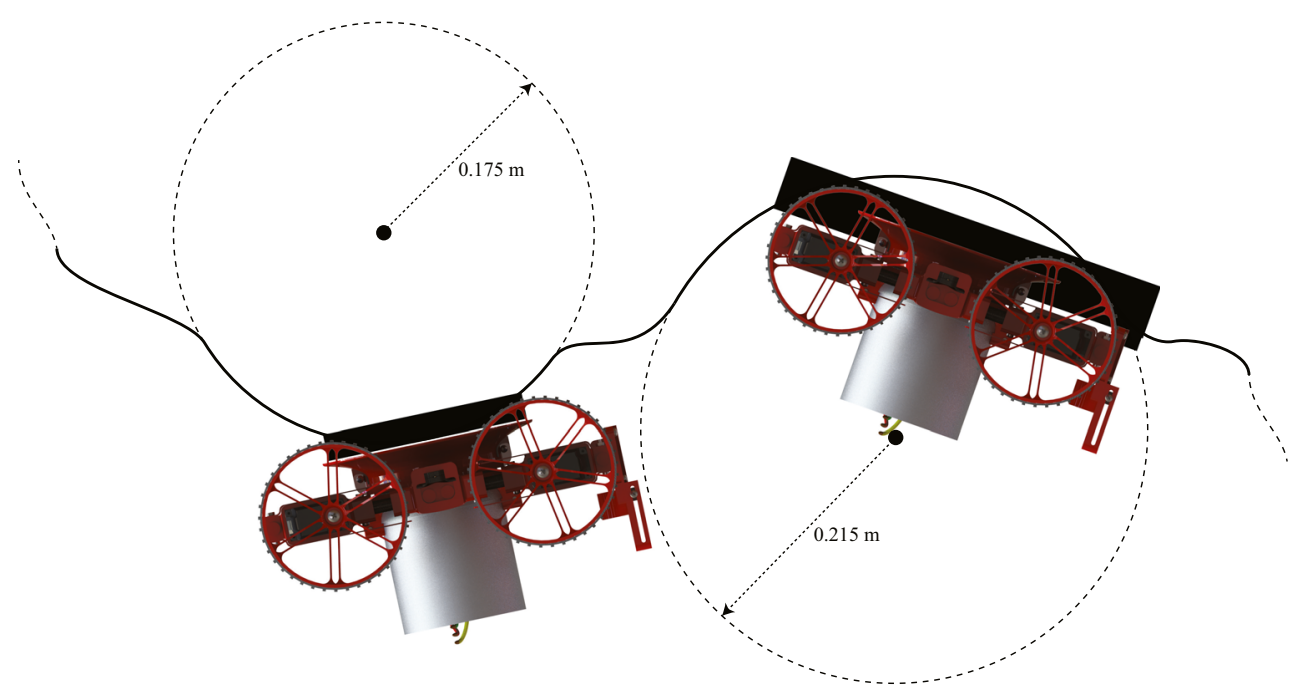

generated force (measured at approximately $38 \mathrm{~N}$ ), which is accounted as the worst adhesion case due to loss of optimal shroud-surface distance, is still larger than the maximal critical force $(33.5 \mathrm{~N})$ required at 129 and 231 degree inclinations [24], which ensures that the presented robot configuration will remain successfully adhered and mobile.

In addition, the minimum concave case will also be affected by the dimensions of the scanning equipment mounted on the sides the robot chassis, which could increase its outer dimensions and limit the minimum supported negative curvatures. For addressing this issue, the proposed climbing robot design possesses the advantage of utilizing a modularized approach, where all VA and wheel segments can be adjusted prior to a mission according to surface specifications and payload requirements. In this way and for known target surfaces and payload, the VA can be placed at the optimal distance according to the surface geometry, the wheels can be set further close or apart from each other, while the shroud can be interchanged to address such changes in wheel distances, thus providing a customized design for achieving robot operation that is tailored to set payload and surface requirements.

Furthermore, robot orientation changes on curved surfaces create the challenge of wheel-surface contact that can affect the platform's mobility, given that turning motions over curvature cases can only lead to three-wheel to surface tangency and reduction of friction forces. The effect of wheel to surface contact over curved surfaces can be reduced given definition of path planning algorithms like the sawtooth-based case proposed in this article, which handle the area coverage missions by constraining the robot heading. Lastly, under inspection-related applications it is important that the surface tolerances and maximum supported adhesion forces are be considered and inserted in the control algorithm, for ensuring the Non-Destructive Testing (NDT) compliance of the robot equipment. In the case of the proposed EMPC control scheme and the target application of aircraft fuselage inspection, the maximal force that the structure of the aircraft could tolerate would be inserted as a constraint on the maximum total force applied on the surface, measured from the modified (3), thus giving the new constraint $0<F_{\max }<F_{\max }^{+}$. It is important to note that throughout the project CompInnova, NDT compliance tests have been verified on composite aircraft fuselage panels, where the VA was operating at full adhesion capacity on pre-scanned damaged and non-damaged areas, where the post-scan results showed no new damages created, while no propagation of existing damages was identified.

Alternative robot designs, adhesion controllers and path planning algorithms for addressing the complex cases of unknown curved surfaces under random orientations are current work in progress. Further more, the next milestone is the design of a generalized model based control framework that enables the fusion of geometrical, motion, and slippage information for dynamic adhesion control, taking into consideration the force and torque model for 2DOF inclination changes. Cases considering the robot under payload and CoM variations during the operation and task execution on real airplane fuselages, are also parts of future work.

Author Contributions Andreas Papadimitriou: Methodology, software, validation, formal analysis, investigation, data curation, writingoriginal draft preparation, writing-review and editing, visualization.

George Andrikopoulos: Conceptualization, visualization, writingoriginal draft preparation, writing-review and editing, software, project administration, supervision, resources.

George Nikolakopoulos: Methodology, project administration, supervision, resources.

Funding Open access funding provided by Lulea University of Technology. This work has received funding from the European Union's H2020 Framework Programme under the call FET-OPEN, grant agreement No. 665238. 


\section{Declarations}

Conflict of interest/Competing interests The authors declare that they have no conflict of interest.

Open Access This article is licensed under a Creative Commons Attribution 4.0 International License, which permits use, sharing, adaptation, distribution and reproduction in any medium or format, as long as you give appropriate credit to the original author(s) and the source, provide a link to the Creative Commons licence, and indicate if changes were made. The images or other third party material in this article are included in the article's Creative Commons licence, unless indicated otherwise in a credit line to the material. If material is not included in the article's Creative Commons licence and your intended use is not permitted by statutory regulation or exceeds the permitted use, you will need to obtain permission directly from the copyright holder. To view a copy of this licence, visit http://creativecommons. org/licenses/by/4.0/

\section{References}

1. Xiao, J., Li, B., Ushiroda, K., Song, Q.: Rise-Rover: A wallclimbing robot with high reliability and load-carrying capacity. 2015 IEEE International Conference on Robotics and Biomimetics, IEEE-ROBIO 2015, pp. 2072-2077. https://doi.org/10.1109/ ROBIO.2015.7419079 (2015)

2. Raju, D., Dethe, S.B.J.: Developments in Wall Climbing Robots: A Review. Int. J. Eng. Res. Gen. Sci. 2 (2014)

3. Shang, J., Sattar, T., Chen, S., Bridge, B.: Design of a climbing robot for inspecting aircraft wings and fuselage. Ind. Robot An Int. J. 34(6), 495-502 (2007). https://doi.org/10.1108/ 01439910710832093

4. Alkalla, M.G., Fanni, M.A., Mohamed, A.: Versatile Climbing Robot for Vessels Inspection. Int. Conf. Control. Autom. Robot., 18-23 (2015)

5. Lu, X., Zhao, S., Yu, D., Liu, X.: Pylon-Climber: A novel climbing assistive robot for pylon maintenance. Ind. Robot. 44(1), 38-48 (2017). https://doi.org/10.1108/IR-06-2016-0172

6. Jianzhong, S.: Development of a climbing robot for inspection of long weld lines. Ind. Robot: Int. J. 35(3), 217-223 (2008). https://doi.org/10.1108/01439910810868534

7. Yoshida, Y., Ma, S.: Design of a Wall-Climbing Robot with Passive Suction Cups. 2010 IEEE International Conference on Robotics and Biomimetics, 1513-1518. https://doi.org/10.1109/ ROBIO.2010.5723554, http://ieeexplore.ieee.org/lpdocs/epic03/ wrapper.htm?arnumber $=5723554$ (2010)

8. Thanh Nguyen, S., La, H.M.: Development of a Steel Bridge Climbing Robot. arXiv:1803.08209 (2018)

9. Espinoza, R.V., de Oliveira, A.S., de Arruda, L.V.R., Junior, F.N.: Navigation's stabilization system of a magnetic adherencebased climbing robot. J. Intell. Robot. Syst. 78(1), 65-81 (2015). https://doi.org/10.1007/s10846-014-0076-Z

10. Wang, C., Gu, J., Li, Z.: Switching motion control of the climbing robot for aircraft skin inspection. In: 2019 IEEE International Conference on Fuzzy Systems (FUZZ-IEEE), pp. 1-6 (2019)

11. Hagiwara, D., Amakawa, T., Yamada, Y., Nakamura, T.: Traveling-wave-type wall-climbing robot for airplane surface inspection. In: International Conference on Climbing and Walking Robots and the Support Technologies for Mobile Machines CLAWAR 2018, vol. 1, pp. 315-323 (2018)

12. Ramalingam, B., Manuel, V.-H., Elara, M.R., Vengadesh, A., Lakshmanan, A.K., Ilyas, M., James, T.J.Y.: Visual inspection of the aircraft surface using a teleoperated reconfigurable climbing robot and enhanced deep learning technique. Int. J. Aerosp. Eng. 2019, 5137139 (2019). https://doi.org/10.1155/2019/5137139

13. Schleupen, J., Engemann, H., Bagheri, M., Kallweit, S., Dahmann, P.: Developing a climbing maintenance robot for tower and rotor blade service of wind turbines. In: Rodić, A., Borangiu, T. (eds.) Advances in Robot Design and Intelligent Control, pp. 310-319. Springer International Publishing, Cham (2017)

14. Alkalla, M.G., Fanni, M.A., Mohamed, A.M., Hashimoto, S.: Tele-operated propeller-type climbing robot for inspection of petrochemical vessels. Ind. Robot. https://doi.org/10.1108/IR-072016-0182 (2017)

15. Ahmed, M., Eich, M., Bernhard, F.: Design and control of mira: A lightweight climbing robot for ship inspection. In: ILCPA Volume 55, International Letters of Chemistry, Physics and Astronomy, vol. 55, pp. 128-135. SciPress Ltd (2015)

16. Papadimitriou, A., Andrikopoulos, G., Nikolakopoulos, G.: Development and control of a differential wall climbing robot based on vortex adhesion. In: 2019 18th European Control Conference (ECC), pp. 1610-1615 (2019)

17. Shujah, A., Habib, H., Shaikh, S., Ishfaq, A.R., Tahir, H., Iqbal, J.: Design and implementation of semi-autonomous wall climbing robot using vacuum suction adhesion. In: 2019 IEEE 17th World Symposium on Applied Machine Intelligence and Informatics (SAMI), pp. 275-280 (2019)

18. Li, B., Ushiroda, K., Yang, L., Song, Q., Xiao, J.: Wall-climbing robot for non-destructive evaluation using impact-echo and metric learning SVM. Int. J. Intell. Robot. Appl. 1(3), 255-270 (2017). https://doi.org/10.1007/s41315-017-0028-4

19. Muthugala, V., Vega-Heredia, M., Mohan, R.E., Vishaal, S.: Design and control of a wall cleaning robot with adhesionawareness. Symmetry 12, 122 (2020). https://doi.org/10.3390/ sym 12010122

20. Shi, K., Li, X.: Vacuum suction unit based on the zero pressure difference method. Phys. Fluids 32(1), 017104 (2020). https://doi.org/10.1063/1.5129958

21. Hillenbrand, C., Schmidt, D., Berns, K.: CROMSCI: development of a climbing robot with negative pressure adhesion for inspections. Ind. Robot An Int. J. 35(3), 228-237 (2008). https://doi.org/10.1108/01439910810868552

22. Kawasaki, S., Kikuchi, K.: Development of a Small Legged Wall Climbing Robot with Passive Suction Cups. xIcdes2014, 112-116 (2014)

23. Andrikopoulos, G., Nikolakopoulos, G.: Vortex actuation via electric ducted fans: an experimental study. J. Intell. Robot. Syst. 95(3), 955-973 (2019). https://doi.org/10.1007/s10846018-0925-2

24. Andrikopoulos, G., Papadimitriou, A., Nikolakopoulos, G.: On Model-based Adhesion Control of a Vortex Climbing Robot. In: Conference: IEEE/RSJ International Conference on Intelligent Robots and Systems (IROS), The Venetian Macao, Macau, China (2019)

25. Qin, S.J., Badgwell, T.A.: A survey of industrial model predictive control technology. Control. Eng. Pract. https://doi.org/10.1016/ S0967-0661(02)00186-7 (2003)

26. Kayacan, E., Ramon, H., Saeys, W.: Robust trajectory tracking error model-based predictive control for unmanned ground vehicles. IEEE/ASME Trans. Mechatron. https://doi.org/10.1109/ TMECH.2015.2492984 (2016)

27. Li, Z., Yang, C., Su, C.Y., Deng, J., Zhang, W.: VisionBased Model Predictive Control for Steering of a Nonholonomic Mobile Robot. IEEE Trans. Control Syst. Technol. https://doi.org/10.1109/TCST.2015.2454484 (2016)

28. Andrade, R., Raffo, G.V., Normey-Rico, J.E.: Model predictive control of a tilt-rotor UAV for load transportation. In: 2016 European Control Conference, ECC 2016 (2016) 
29. Takács, B., Števek, J., Valo, R., Kvasnica, M.: Python code generation for explicit MPC in MPT. In: 2016 European Control Conference, ECC 2016, pp. 1328-1333 (2017)

30. Herceg, M., Kvasnica, M., Jones, C.N., Morari, M.: Multiparametric toolbox 3.0. In: 2013 European Control Conference, ECC 2013 (2013)

31. Corke, P.I.: Robotics, vision \& control: Fundamental algorithms in MATLAB, 2 edn. Springer. ISBN 978-3-319-54413-7 (2017)

32. Ljung, L.: Ljung L System Identification Theory for User.pdf. vol. 25. http://linkinghub.elsevier.com/retrieve/pii/0005109889900198 (1987)

33. Bemporad, A., Morari, M., Dua, V., Pistikopoulos, E.N.: The explicit linear quadratic regulator for constrained systems. Automatica 38(1), 3-20 (2002). https://doi.org/10.1016/S00051098(01)00174-1

34. Brusell, A., Andrikopoulos, G., Nikolakopoulos, G.: Novel considerations on the negative pressure adhesion of electric ducted fans: An experimental study. 2017 25th Mediterranean Conference on Control and Automation, MED 2017, pp. 14041409. https://doi.org/10.1109/MED.2017.7984315 (2017)

35. Andrikopoulos, G., Nikolakopoulos, G., Manesis, S.: An Experimental Study on Thermodynamic Properties of Pneumatic Artificial Muscles. In: Andrikopoulos, G., Nikolakopoulos, G., Manesis, S. (eds.) 20th Mediterranean Conference on Control and Automation (MED), pp. 1334-1340, Barcelona (2012)

36. The Mathworks, Inc., Natick, Massachusetts: MATLAB and Simulink version 9.6.0.1072779 (R2019a) (2019)

37. Vicon, Inc, Oxford, United Kingdom: Vicon Motion Systems. https://www.vicon.com (2021)

Publisher's Note Springer Nature remains neutral with regard to jurisdictional claims in published maps and institutional affiliations.

Andreas Papadimitriou is currently a Ph.D. candidate in the Robotics \& AI Group at Luleå University of Technology, Sweden. His research interests are focused on the control of robotic applications for inspection and repair purposes.

George Andrikopoulos is an Assistant Professor at the Mechatronics and Embedded Control Systems Unit of the Royal Institute of Technology (KTH) in Stockholm, Sweden. His research interests are focused on the design, development and control of autonomous robotic systems via the use of soft and compliant actuators, climbing robotic solutions for industrial inspection, exoskeletons for medical purposes, and home service robotics. He has been involved in the creation, technical implementation, scientific or overall management of several European and National R\&D projects on biorobotics, inspection and industrial robotics, including H2020 FETOPEN CSA "VORTEX", H2020 FETOPEN RIA "COMPINNOVA", H2020 RIA ICT "AEROWORKS", H2020 IA CE "SIMS" and Vetenskapsrådet (Swedish Research Council) "BAHRT". He has also initiated multiple national research collaborations funded by Swedish Universities and Colleges, as well 2 as an international collaboration with HONDA Research Institute Japan. His scientific work includes more than 50 articles published in high-impact International Journals and Conferences in the fields of interest, while his work has been listed in the TOP 25 most popular publications in Elsevier's Control Engineering Practice. He has served as an IPC member and Technical Track Chair for multiple international conferences, while he currently serves a topic editor for MDPI Actuators. He has been an active reviewer in several highimpact international journals and conferences, a co-founding member of the Socially Intelligent Robotics Consortium, a member of the Swedish Society of Biomechanics and a member of IEEE (CSS, RAS, IES, Young Professionals).
George Nikolakopoulos is a full Professor on Robotics and Automation at the Department of Computer Science, Electrical and Space Engineering at Luleå University of Technology, Luleå, Sweden. He is also a JPL/NASA research affiliated working in the SubT competition of NASA in the field of aerial subterranean exploration of planets. His work is focusing in the area of Robotics, Control Applications and AI Systems, while he has a significantly large experience in Creating and Managing European and National Research Projects. In the past he has been working as project manager and principal investigator in Several R\&D\&I projects funded by the EU, ESA, Swedish and the Greek National Ministry of Research, with some examples to be: a) EU funded projects: FLEXA (IP), C@R (IP), NANOMA (STREP), SYMBIOSISEU (STREP), CONFIDENCE (STREP), PROMOVEO (STREP), CommRob (STREP), b) ARTEMIS funded projects: R5-COP, and c) Swedish funded projects: Mine Patrolling Rovers, Pneumatic Muscle Dancer, etc. Prof. Nikolakopoulos was also the coordinator of the successful projects AEROWORKS and DISIRE, while in 2019, has been included in the list of Top100 Innovators in Sweden. Beginning 2015 he was granted the FETOPEN H2020 project CompInnova in the field of airplane inspection by advanced inspection methodologies and robotics. In 2014 he was granted as coordinator the H2020-ICT AEROWORKS project in the field of Aerial Collaborative UAVs and the H2020-SPIRE project DISIRE in the field of Integrated Process Control of a total budget of 12M Euros. In 2013 he received a grant of $220 \mathrm{~K}$ Euros for establishing the bigger outdoors motion capture system in Sweden and most probably in Europe as part of the FROST Field Robotics Lab at Luleå University of Technology. In 2014, he was nominated as LTU's Wallenberg candidate, one out of three nominations from the university and 16 in total engineering nominations in Sweden. In year 2003, he received the Information Societies Technologies (IST) Prize Award for the best paper that Promotes the scopes of the European IST (currently known as ICT) sector. His publications in the field of UAVs and AI have received top recognition from the related scientific community, while have been several times listed in the TOP 25 most popular publications in Control Engineering Practice from Elsevier. In 2014 he received the 2014 Premium Award for Best Paper in IET Control Theory and Applications, Elsevier for outstanding research work in the area of UAVs, This is a Premium Award to recognise the best research papers published during the last two years in this Journal. His published scientific work includes more than 200 published International Journals and Conferences in the fields of interest. Moreover, he has served as IPC member and Technical Track Chair for multiple international conferences and have been Associate Editor and Reviewer of Several International Journals and Conferences. Prof. Nikolakopoulos works also as a member of the ARTEMIS scientific Council in the European Commission and he is co-leading the Strong Area of AI Research at LTU in the field of Applied AI and AI-DIH. 